\title{
A "Back Light System" for Identification of Sites for Endoscopic Ultrasound-Guided Fine-Needle Aspiration in Solid Pancreatic Masses: A Prospective, Randomized Study with a Crossover Design
}

\author{
Ryo Harada', Hironari Kato ${ }^{1}$, Soichiro Fushimi ${ }^{2}$, Hirofumi Inoue ${ }^{2}$, Daisuke Uchida ${ }^{1}$, Yutaka Akimoto ${ }^{1}$, Takeshi Tomoda', \\ Kazuyuki Matsumoto ${ }^{1}$, Yasuhiro Noma ${ }^{1}$, Naoki Yamamoto ${ }^{1}$, Shigeru Horiguchi ${ }^{1}$, Koichiro Tsutsumi ${ }^{1}$ and Hiroyuki Okada ${ }^{1}$ \\ ${ }^{1}$ Department of Gastroenterology and Hepatology, Okayama University Graduate School of Medicine, Dentistry, and Pharmaceutical \\ Sciences, Okayama, ${ }^{2}$ Department of Pathology, Okayama University Hospital, Okayama, Japan
}

Background/Aims: We applied a back light system (BLS) with a magnifying glass to improve the ability to assess the adequacy of specimen sampling using endosonography. We conducted this study to evaluate the efficacy of the BLS in sampling of specimens by endoscopic ultrasound-guided fine needle aspiration of solid pancreatic masses.

Methods: This was a prospective, randomized, crossover, single-center clinical trial. An endosonographer evaluated adequacy on gross visual inspection and identified whitish specimen sampling sites with and without the BLS according to a randomization sequence in the first and second passes with a 25-G needle. On cytological evaluation, the presence of well-defined pancreatic ductal epithelium was evaluated by a cytopathologist who was blinded to any clinical information.

Results: A total of 80 consecutive patients were eligible during the study period. Adequacy was observed for 52 specimens (65\%) with the BLS and $54(68 \%)$ without the BLS $(p=0.88)$. In assessment of specimen adequacy on gross examination, only fair agreement was observed both with and without BLS (kappa score 0.40 and 0.29 , respectively).

Conclusions: The BLS did not influence the ability to identify specimen sampling sites or reliable assessment of specimen site adequacy using gross visual inspection. Clin Endosc 2019;52:334-339

Key Words: Endosonographer; Cytology; Rapid on-site evaluation; Endoscopic ultrasound-guided fine-needle aspiration; Pancreatic neoplasms

\section{INTRODUCTION}

Endoscopic ultrasound-guided fine-needle aspiration (EUS-FNA) is safe and useful for the diagnosis of pancreatic diseases. ${ }^{1}$ EUS-FNA accuracy is dependent on several factors: the density of cancer cells in the tissue, the endosonographer's

Received: November 22, 2018 Revised: January 7, 2019

Accepted: January 22, 2019

Correspondence: Ryo Harada

Department of Gastroenterology and Hepatology, Okayama University Graduate School of Medicine, Dentistry, and Pharmaceutical Sciences, 2-5-1 Shikata-cho, Kita-ku, Okayama 700-8558, Japan

Tel: +81-86-235-7219, Fax: +81-86-225-5991, E-mail: rharada927@yahoo.co.jp ORCID: https://orcid.org/0000-0002-7213-8661

(c) This is an Open Access article distributed under the terms of the Creative Commons Attribution Non-Commercial License (http://creativecommons.org/ licenses/by-nc/3.0) which permits unrestricted non-commercial use, distribution, and reproduction in any medium, provided the original work is properly cited. skill in performing the procedure, the type and diameter of the needle used, the number of needle passes, the aspiration method, and the method of sample processing. ${ }^{2-10}$ Among these factors, rapid on-site evaluation (ROSE) by an attending cytotechnologist or cytopathologist has the potential to improve the diagnostic yield of EUS-FNA for cytological diagnosis. ${ }^{11-15}$ However, at many institutions, the adequacy of specimens is not always evaluated on-site by a trained cytotechnologist or cytopathologist. Accordingly, ROSE by the endosonographer could be a practical alternative. ROSE by the endosonographer could potentially minimize the number of passes and improve the diagnostic accuracy of EUS-FNA, even if an on-site cytotechnologist or cytopathologist is not routinely available. ${ }^{16}$

To improve the endosonographer's ability to detect adequate specimen sampling sites, we used a back light system 
(BLS) with a magnifying glass, the Bioevaluator ${ }^{\circledR}$ (Murazumi, Himeji, Japan) (Fig. 1), for identification of whitish specimens. ${ }^{17}$ The Bioevaluator ${ }^{\circledR}$ was developed to enable identification of specimen aspiration sites and ROSE using EUS-FNA. It is considered useful for the identification of appropriate tissue sampling sites obscured by blood, even when small. We conducted the present study to determine whether the use of this BLS can improve the results of ROSE on endosonography.

\section{MATERIALS AND METHODS}

\section{Patients}

Patients with pancreatic masses diagnosed with computed tomography or magnetic resonance imaging were prospectively enrolled at Okayama University Hospital (Okayama, Japan) between March 2014 and March 2015. Patients with the following conditions were excluded: a pancreatic mass that was undetectable with EUS; severe ascites; other serious disease such as unstable angina, symptomatic congestive heart failure, or hepatorenal failure; risk of bleeding; or refusal or inability to provide informed consent. The Institutional Re- view Board approved this trial, and written informed consent for voluntary participation was obtained from all patients before they entered the study. This trial was registered in the UMIN Clinical Trials Registry (UMIN000013393).

\section{Randomization}

To control the variables that could influence study results, we randomized the patients using a crossover design for BLS use or non-use in the first and second passes (Fig. 2). Randomization was performed immediately after the endosonographer detected the pancreatic mass. An individual other than the endosonographer performing the EUS-FNA used biased-coin randomization to allocate cases to the groups. Randomization was performed in a 1:1 ratio.

\section{EUS-FNA technique}

EUS-FNA was performed using a curvilinear echoendoscope (GF-UCT 240 or GF-UCT 260; Olympus, Tokyo, Japan) with corresponding display units (Prosound SSD $a-10$; Hitachi Aloka Medical, Tokyo, Japan). EUS procedures were carried out with the patient in the left lateral position under conscious sedation using pethidine hydrochloride and diazepam. EUS-
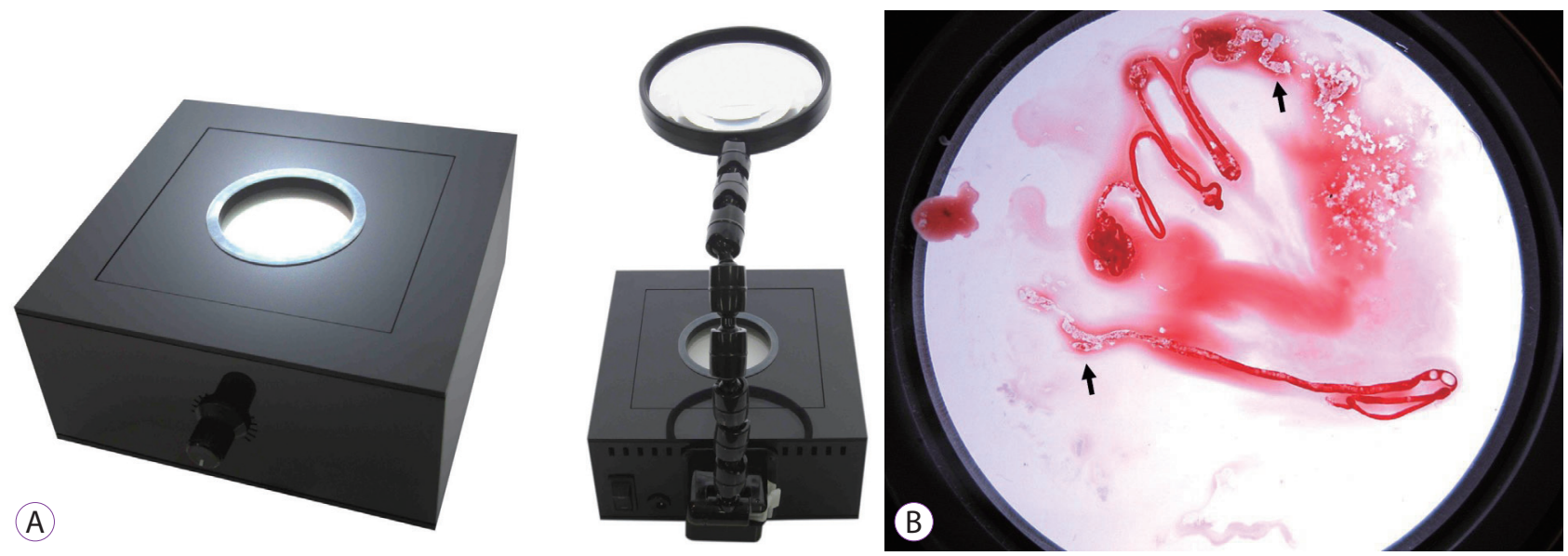

Fig. 1. (A) Back light system with a magnifying glass. (B) The back light system clearly showed white areas in an endoscopic ultrasound-guided fine needle aspiration specimen (arrows).

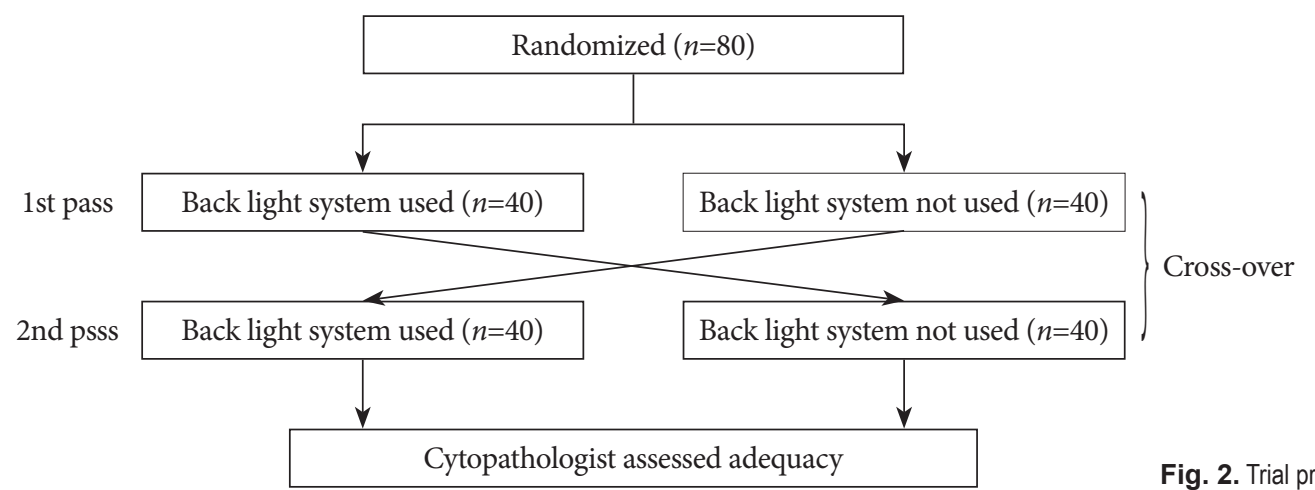

Fig. 2. Trial profile 
FNA was performed with the same $25-\mathrm{G}$ needle (Echotip ${ }^{\circledR}$ or Procore $^{\circledR}$; Cook Medical, Bloomington, IN, USA, SonoTip Pro Control $^{\circledR}$; Medi-Globe, Achenmühle, Germany, or Expect ${ }^{\circledR}$; Boston Scientific, Natick, MA, USA) in the first and second passes. We choose a 25-G needle for flexibility of access, even with a transduodenal approach. After the second pass, the endosonographer performed EUS-FNA with a 25-, 22-, 21-, or 19-G needle (Echotip ${ }^{\circledR}$; Procore ${ }^{\circledR}$; SonoTip Pro Control ${ }^{\circledR}$; Expect $^{\circledR}$; or an EUS SONOPSY CY ${ }^{\mathbb{R}}$; Hakko, Nagano, Japan). The choice of needle was based on the operator's preference to achieve the safest and most successful puncture. Suction was applied with a $10-\mathrm{mL}$ or $20-\mathrm{mL}$ syringe while the needle was moved 20 times per needle pass within the lesion. The needle pass was repeated until enough specimens were obtained, with a minimum of 2 and a maximum of 6 passes. Adverse events were defined based on a lexicon for endoscopic adverse events described by the American Society Gastrointestinal Endoscopy. ${ }^{18}$

\section{Bioevaluator $^{\circledR}$}

The Bioevaluator ${ }^{\circledR}$ was developed by Murazumi Industrial Co. Ltd. in collaboration with one of the authors (HI) to identify the aspiration site during EUS-FNA for ROSE (Fig. 1). This device costs about $\$ 1,800$ and illuminates samples from below, using a $12-\mathrm{V}$ light-emitting diode. The aspirated materials are expressed onto a glass plate and illuminated. We can easily identify appropriate tissue, even when obscured by blood.

\section{Specimen processing}

Specimen processing was performed by one of 8 endosonographers (HK, DU, YA, TT, KM, NY, SH and KT) with different levels of EUS-FNA experience (4 endosonographers had experience with less than 100 cases and 4 had experience with more than 100 cases), after reading the manual. None had previous experience with specimen processing. The tissue specimen was expressed onto a glass plate immediately after it was obtained. An endosonographer who did not perform the EUS-FNA then evaluated the adequacy of the specimen on gross examination, and identified whitish specimens with or without use of the BLS using forceps according to the randomization sequence.

A trained cytotechnologist also picked up specimens with the BLS from the first pass after the endosonographer did. The cytotechnologist immediately evaluated the specimen to ensure adequacy. The specimen was smeared onto glass slides. One slide was air dried and stained with Hemacolor ${ }^{\circledR}$ (Merck, Darmstadt, Germany). Another slide was stained with alcohol. After each EUS-FNA procedure, a cytotechnologist performed Papanicolaou staining. The remaining specimens were fixed in $10 \%$ buffered neutral formalin and embedded in paraffin for hematoxylin and eosin staining, and evaluated histologically.

\section{Cytological evaluation}

On cytological evaluation, the presence of clusters of pancreatic ductal epithelium in both Hemacolor and Papanicolaou stained slides was evaluated by a cytopathologist (SF) who was blinded to any clinical information and BLS use. A similar evaluation form that required a grade assignment for adequacy ( 1 , adequate; 2 , equivocal; or 3 , inadequate) was completed for each slide based on microscopic assessment. The definition of adequacy was determined by the presence of pancreatic ductal epithelium: 1 (adequate), $>10$ cell clusters consisting of 50 cells, or 40 cells in a high-power field; 2 (equivocal), not showing the criteria for grades 1 and 3; and 3 (inadequate), cellular quality inadequate for diagnosis.

\section{Final diagnosis}

The final diagnosis was made according to one of the following reference methods: (1) a histological diagnosis based on a surgically resected specimen; (2) a positive FNA diagnosis for malignancy in patients with unresectable tumors and compatible clinical follow-up; or (3) a negative FNA diagnosis for malignancy and a minimum clinical follow-up time of 6 months.

\section{Statistical analysis}

The primary outcome was the adequacy of slides with and without BLS use. The secondary outcome was the gross visual assessment of specimen adequacy. The relevant risk factors in BLS use and non-use groups were assessed with Fisher's exact test for categorical variables. We used simple kappa coefficients to evaluate agreement on gross examination of specimens between the endosonographer and cytopathologist. Kappa $=1$ indicates perfect agreement, with kappa $=-1$ indicating complete disagreement. Probability values $<0.05$ were considered significant. Statistical analyses were performed with the software package JMP ver. 12.2.0 (SAS, Cary, NC, USA).

\section{RESULTS}

\section{Characteristics of the patients and procedures}

Between March 2014 and March 2015, 80 consecutive patients with pancreatic masses diagnosed with computed tomography or magnetic resonance imaging were identified. All accepted our invitation to participate in the study. These patients were randomized to BLS use and non-use groups (Fig. 2).

The baseline characteristics are summarized in Table 1. Me- 
dian age was 67 years (20-90 years); there were 30 females and 50 males. In terms of location, 38 (48\%) of tumors were found in the pancreatic head, 25 (31\%) in the pancreatic body, and 17 (21\%) in the pancreatic tail. The median size of lesions was $27 \mathrm{~mm}(8-102 \mathrm{~mm})$ in the long axis and $20 \mathrm{~mm}(6-96 \mathrm{~mm})$ in the short axis. All 80 patients underwent EUS-FNA. A me-

Table 1. Patients and Lesion Characteristics

\begin{tabular}{|c|c|}
\hline Characteristic & Value \\
\hline No. of patients & 80 \\
\hline Age, yr; median (IQR) & $68(60-73)$ \\
\hline Sex (male/female) & $50 / 30$ \\
\hline BMI, median (IQR) & $21.1(19.0-22.8)$ \\
\hline ASA classification (1/2) & $31 / 49$ \\
\hline Location (head/body/tail) & $38 / 25 / 17$ \\
\hline Long axis, mm; median (IQR) & $27(17-38)$ \\
\hline Short axis, mm; median (IQR) & $20(15-27)$ \\
\hline \multicolumn{2}{|c|}{$\begin{array}{l}\text { Endoscopic ultrasound-guided fine needle aspiration } \\
\text { (per lesion) }\end{array}$} \\
\hline Feasibility, $n(\%)$ & $80(100)$ \\
\hline No. of passes, median (IQR) & $3(2-4)$ \\
\hline \multicolumn{2}{|l|}{ Fine needle aspiration site, $n(\%)$} \\
\hline Stomach & $45(56)$ \\
\hline Duodenum & $35(44)$ \\
\hline
\end{tabular}

ASA, American Society of Anesthesiologists; BMI, body mass index; IQR, interquartile range. dian of 3 FNA passes (2-6) per patient was performed on the pancreatic mass. EUS-FNA was performed via the stomach in $45(56 \%)$ patients and via the duodenum in 35 (44\%).

\section{Adequacy rate in the BLS and non-BLS groups}

The 1st and 2nd passes in 80 patients yielded a total of 160 slides. In the BLS group, 24 of the 40 slide specimens (60\%) were assessed as adequate in the 1st pass, and 28 of 40 (70\%) assessed as adequate in the 2 nd pass. In the non-BLS group, 27 of 40 specimens $(67.5 \%)$ were assessed as adequate in the 1 st pass, and 27 of $40(67.5 \%)$ assessed as adequate in the 2 nd pass. Thus, 52 specimens (65\%) underwent adequacy assessment in the BLS group and 54 (67.5\%) underwent assessment in the non-BLS group, with no significant difference $(p=0.88)$ (Table 2).

\section{Gross visual assessments of specimen adequacy}

We used the kappa coefficient (scale of -1 to 1 ) to determine the chance-corrected level of agreement between each grader and the criterion standard (microscopic assessment by the cytopathologist). The kappa score for the endosonographer with use of the BLS was 0.40 (95\% confidence interval [CI], 0.17-0.62). The kappa score without use of the BLS was 0.29 (95\% CI, 0.10-0.48). These data indicate only fair agreement with the final cytopathology interpretation (Table 3). There was no significant difference in accuracy between the use of $\operatorname{BLS}(61 / 80$ [76\%]) and non-use of BLS (53/80 [66\%]) $(p=0.22)$.

Table 2. The Microscopic Adequacy Rate of the Slides, with and without the Use of the Back Light System

\begin{tabular}{|c|c|c|c|c|c|c|}
\hline & \multicolumn{3}{|c|}{ Back light system used } & \multicolumn{3}{|c|}{ Back light system not used } \\
\hline & 1st pass & 2nd pass & Total & 1st pass & 2nd pass & Total \\
\hline Adequate & 24 & 28 & 52 & 27 & 27 & 54 \\
\hline Inadequate & 16 & 12 & 28 & 13 & 13 & 26 \\
\hline
\end{tabular}

Table 3. The Gross Visual Assessments of Specimen Adequacy

(A)

\begin{tabular}{lccc}
\hline & \multicolumn{2}{c}{ Adequate by cytopathology } & \multirow{2}{*}{ Total } \\
\cline { 2 - 3 } & Yes & No & \\
\hline Yes & 49 & 10 & 59 \\
No & 9 & 12 & 21 \\
Total & 58 & 22 & 80 \\
Kappa coefficient & & & 0.40 \\
\hline
\end{tabular}

Predicted to be adequate by an endosonographer with the use of the back light system.
(B)

\begin{tabular}{lccc} 
& \multicolumn{2}{c}{ Adequate by cytopathology } & \multirow{2}{*}{ Total } \\
\cline { 2 - 3 } & Yes & No & \\
\hline Yes & 39 & 5 & 44 \\
No & 22 & 14 & 36 \\
Total & 61 & 19 & 80 \\
Kappa coefficient & & & 0.29 \\
\hline
\end{tabular}

Predicted to be adequate by an endosonographer without the use of the back light system. 


\section{Diagnostic yield of EUS-FNA}

The overall diagnostic yield including both cytologic and histologic result for malignancy in EUS-FNA showed the following: sensitivity, $98.7 \%$; specificity, $100 \%$; positive predictive value, $100 \%$; negative predictive value, $83.3 \%$; and accuracy, 98.8\% (Table 4). The histological diagnostic accuracy for malignancy was $70 \%$ (56/80).

\section{Final diagnoses}

The final diagnoses were pancreatic ductal adenocarcinoma ( $n=63,78.8 \%$ ), neuroendocrine tumor $(n=5,6.3 \%)$, solid pseudopapillary neoplasm $(n=4,5 \%)$, metastatic pancreatic cancer $(n=3,3.8 \%)$, chronic pancreatitis $(n=4,5 \%)$, and autoimmune pancreatitis $(n=1,1.2 \%)$. The final diagnoses were obtained using clinical follow-up in 47 patients (59\%), and surgery in 33 (41\%).

\section{Complications}

There was no bleeding or perforation with EUS-FNA in this series. Mild pancreatitis occurred in 1 patient with pancreatic head cancer; however, this resolved with conservative therapy and prolonged the hospital stay by only 1 night.

\section{DISCUSSION}

Guidelines recommend obtaining 5-7 needle passes for patients undergoing EUS-FNA for solid pancreatic masses. ${ }^{19}$ However, unnecessary punctures may increase the risk of procedure-related complications such as pancreatitis, site infection, bleeding, and tumor seeding. ${ }^{20-22}$ Although ROSE has the potential to improve diagnostic performance and avoid unnecessary punctures, ${ }^{11-15}$ many institutions do not have an onsite cytotechnologist or cytopathologist and the adequacy of specimens is not routinely evaluated. In these institutions, we reported that an intensive, 2-h interactive training program for endosonographers significantly improved their ability to judge specimen sampling site adequacy, as well as their ability to make the correct diagnosis. ${ }^{23}$ Before judging specimen adequacy, we must obtain appropriate specimens. This is more important during ROSE. Thus, we consider the Bioevaluator ${ }^{\circledR}$ BLS useful for identification of whitish specimens because of the visibility provided, especially when using a $25-\mathrm{G}$ needle.
No significant difference in the adequacy of specimens with and without use of the BLS was revealed, for several possible reasons. First, when it was clearly visible, the endosonographer was able to immediately identify the sampling site, even without the aid of the BLS. Second, even with use of the BLS, necrotic or mucin-rich specimens could not be sufficiently distinguished from well-defined pancreatic ductal epithelium. Third, when the endosonographer was able to identify a small specimen sampling site with the BLS, a lack of experience at using forceps interfered with retrieval. The Bioevaluator ${ }^{\circledR}$ is optimized to identify small fragments that are obscured by a blood clot, and thus might be useful for obtaining specimens sufficient for histological diagnosis after skill at retrieving fragments is achieved.

In our subgroup analysis of the gross visual assessment of specimen adequacy, only fair agreement between observers was observed. The kappa coefficient was slightly greater in the BLS group, but there was no significant between-group difference. Iwashita et al. showed the efficacy of macroscopic on-site quality evaluation in estimating the adequacy of histologic core specimens obtained with EUS-FNA using a 19-G needle for solid lesions. ${ }^{24}$ However, it is generally difficult for a 19-G needle to access a transduodenal approach due to needle rigidity. We therefore chose a $25-\mathrm{G}$ needle, which can even access a transduodenal approach.

Nguyen et al. showed that neither trained endosonographers nor cytotechnologists were able to provide a reliable assessment of pancreatic mass EUS-FNA adequacy by performing a gross visual inspection of the specimen; ${ }^{25}$ the kappa coefficient for the endosonographers was 0.19 (95\% CI, 0.080.30 ). Our data indicate better results, but our findings and those reported by Nguyen et al. cannot be compared because of the differences in patient backgrounds and the method by which the specimens were processed. ${ }^{25}$ Matsumoto et al. showed good agreement with a target sample check illuminator (TSCI) ${ }^{26}$ The TSCI can use the 605-nm wavelength, which may account for differences.

The current study has several limitations. First, this was a preliminary study. Therefore, sample size calculation was not done and the sample size might be small. Second, the targets may have been too easy to compare. When many specimens were obtained with EUS-FNA, it was very easy to identify specimen sampling site adequacy, even without the BLS. The

Table 4. The Diagnostic Yields of the Endoscopic Ultrasound-Guided Fine Needle Aspiration

\begin{tabular}{lcccc}
\hline Sensitivity (\%) & Specificity (\%) & PPV (\%) & NPV (\%) & Accuracy (\%) \\
\hline 98.7 & 100 & 100 & 83.3 & 98.8 \\
$74 / 75$ & $5 / 5$ & $74 / 74$ & $5 / 6$ & $79 / 80$ \\
\hline
\end{tabular}

NPV, negative predictive value; PPV, positive predictive value. 
BLS may be useful in difficult cases.

In conclusion, our analyses demonstrated that the BLS did not influence the endosonographer's ability to identify specimen sampling site adequacy or the reliability of gross visual assessment. Trained cytotechnologists or cytologists may use a BLS efficiently. Improvements in the BLS are needed, including identification of the appropriate wavelength, as in TSCI, or use of artificial intelligence to compensate for the lack of routine on-site evaluation by a cytotechnologist or cytopathologist.

\section{Conflicts of Interest}

Hirofumi Inoue collaborated with Murazumi Industrial Co. Ltd., but had no patent rights or financial relationship. Other authors have no financial conflicts of interest.

\section{Author Contributions}

Conceptualization: Ryo Harada, Hironari Kato, Hiroyuki Okada

Data curation: RH, Yutaka Akimoto, Shigeru Horiguchi

Investigation: Daisuke Uchida, YA, Kazuyuki Matsumoto, Yasuhiro Noma, Naoki Yamamoto, SH, Koichiro Tsutsumi

Methodology: Soichiro Fushimi, Hirofumi Inoue

Project administration: SF, HI

Writing-original draft: $\mathrm{RH}$

Writing-review\&editing: RH, HK, SF, Takeshi Tomoda

\section{REFERENCES}

1. Vilmann P, Jacobsen GK, Henriksen FW, Hancke S. Endoscopic ultrasonography with guided fine needle aspiration biopsy in pancreatic disease. Gastrointest Endosc 1992;38:172-173.

2. Erickson RA, Sayage-Rabie L, Beissner RS. Factors predicting the number of EUS-guided fine-needle passes for diagnosis of pancreatic malignancies. Gastrointest Endosc 2000;51:184-190.

3. Kim J, Ryu JK, Park JM, et al. Clinical factors associated with accuracy of EUS-FNA for pancreatic or peripancreatic solid mass without on-site cytopathologists. J Gastroenterol Hepatol 2014;29:887-892.

4. Ishiwatari H, Hayashi T, Kawakami H, et al. Randomized trial comparing a side-port needle and standard needle for EUS-guided histology of pancreatic lesions. Gastrointest Endosc 2016;84:670-678.

5. Siddiqui UD, Rossi F, Rosenthal LS, Padda MS, Murali-Dharan V, Aslanian HR. EUS-guided FNA of solid pancreatic masses: a prospective, randomized trial comparing 22-gauge and 25-gauge needles. Gastrointest Endosc 2009;70:1093-1097.

6. Carrara S, Anderloni A, Jovani M, et al. A prospective randomized study comparing $25-\mathrm{G}$ and $22-\mathrm{G}$ needles of a new platform for endoscopic ultrasound-guided fine needle aspiration of solid masses. Dig Liver Dis 2016;48:49-54.

7. Mohamadnejad M, Mullady D, Early DS, et al. Increasing number of passes beyond 4 does not increase sensitivity of detection of pancreatic malignancy by endoscopic ultrasound-guided fine-needle aspiration. Clin Gastroenterol Hepatol 2017;15:1071-1078.e2.

8. Attam R, Arain MA, Bloechl SJ, et al. "Wet suction technique (WEST)": a novel way to enhance the quality of EUS-FNA aspirate. Results of a prospective, single-blind, randomized, controlled trial using a 22-gauge needle for EUS-FNA of solid lesions. Gastrointest Endosc 2015;81:14011407.

9. Chen JY, Ding QY, Lv Y, et al. Slow-pull and different conventional suction techniques in endoscopic ultrasound-guided fine-needle aspiration of pancreatic solid lesions using 22-gauge needles. World J Gastroenterol 2016;22:8790-8797.

10. Hashimoto S, Taguchi H, Higashi M, et al. Diagnostic efficacy of liquid-based cytology for solid pancreatic lesion samples obtained with endoscopic ultrasound-guided fine-needle aspiration: propensity scorematched analysis. Dig Endosc 2017;29:608-616.

11. Klapman JB, Logrono R, Dye CE, Waxman I. Clinical impact of onsite cytopathology interpretation on endoscopic ultrasound-guided fine needle aspiration. Am J Gastroenterol 2003;98:1289-1294.

12. Iglesias-Garcia J, Dominguez-Munoz JE, Abdulkader I, et al. Influence of on-site cytopathology evaluation on the diagnostic accuracy of endoscopic ultrasound-guided fine needle aspiration (EUS-FNA) of solid pancreatic masses. Am J Gastroenterol 2011;106:1705-1710.

13. Haba S, Yamao K, Bhatia V, et al. Diagnostic ability and factors affecting accuracy of endoscopic ultrasound-guided fine needle aspiration for pancreatic solid lesions: Japanese large single center experience. J Gastroenterol 2013;48:973-981.

14. Matynia AP, Schmidt RL, Barraza G, Layfield LJ, Siddiqui AA, Adler DG. Impact of rapid on-site evaluation on the adequacy of endoscopic-ultrasound guided fine-needle aspiration of solid pancreatic lesions: a systematic review and meta-analysis. J Gastroenterol Hepatol 2014;29:697-705.

15. Keswani RN, Krishnan K, Wani S, Keefer L, Komanduri S. Addition of endoscopic ultrasound (EUS)-guided fine needle aspiration and on-site cytology to EUS-guided fine needle biopsy increases procedure time but not diagnostic accuracy. Clin Endosc 2014;47:242-247.

16. Hayashi T, Ishiwatari H, Yoshida M, et al. Rapid on-site evaluation by endosonographer during endoscopic ultrasound-guided fine needle aspiration for pancreatic solid masses. J Gastroenterol Hepatol 2013;28:656-663.

17. Minami D, Takigawa N, Inoue H, Hotta K, Tanimoto M, Kiura K. Rapid on-site evaluation with BIOEVALUATOR ${ }^{\circ}$ during endobronchial ultrasound-guided transbronchial needle aspiration for diagnosing pulmonary and mediastinal diseases. Ann Thorac Med 2014;9:14-17.

18. Cotton PB, Eisen GM, Aabakken L, et al. A lexicon for endoscopic adverse events: report of an ASGE workshop. Gastrointest Endosc 2010;71:446-454.

19. Wani S, Wallace MB, Cohen J, et al. Quality indicators for EUS. Gastrointest Endosc 2015;81:67-80.

20. Katanuma A, Maguchi H, Yane K, et al. Factors predictive of adverse events associated with endoscopic ultrasound-guided fine needle aspiration of pancreatic solid lesions. Dig Dis Sci 2013;58:2093-2099.

21. Minaga K, Kitano M, Yamashita Y. Surgically resected needle tract seeding following endoscopic ultrasound-guided fine-needle aspiration in pancreatic cancer. J Hepatobiliary Pancreat Sci 2015;22:708-709.

22. Tomonari A, Katanuma A, Matsumori T, et al. Resected tumor seeding in stomach wall due to endoscopic ultrasonography-guided fine needle aspiration of pancreatic adenocarcinoma. World J Gastroenterol 2015;21:8458-8461.

23. Harada R, Kato H, Fushimi S, et al. An expanded training program for endosonographers improved self-diagnosed accuracy of endoscopic ultrasound-guided fine-needle aspiration cytology of the pancreas. Scand J Gastroenterol 2014;49:1119-1123.

24. Iwashita T, Yasuda I, Mukai T, et al. Macroscopic on-site quality evaluation of biopsy specimens to improve the diagnostic accuracy during EUS-guided FNA using a 19-gauge needle for solid lesions: a single-center prospective pilot study (MOSE study). Gastrointest Endosc 2015;81:177-185.

25. Nguyen YP, Maple JT, Zhang Q, et al. Reliability of gross visual assessment of specimen adequacy during EUS-guided FNA of pancreatic masses. Gastrointest Endosc 2009;69:1264-1270.

26. Matsumoto K, Ueki M, Takeda Y, et al. Development of a device for detecting target specimens from EUS-guided FNA samples. Endosc Int Open 2015;3:E662-E664. 